\title{
Cryo-SEM Imaging and Analysis of Frozen-Hydrated PEG-AA Microgels
}

\author{
Jing Liang ${ }^{2}$, Feiyue Teng ${ }^{2}$, Matthew Libera ${ }^{2}$, and Tsengming Chou ${ }^{1}$ \\ 1. Laboratory for Multiscale Imaging, Stevens Institute of Technology, Hoboken, NJ USA \\ 2. Dept. of Chemical Engr. \& Materials Science, Stevens Institute of Technology, Hoboken, NJ USA
}

Microgels are microscopic hydrogels with diameters of $\sim 0.1-10 \square \mathrm{m}$. They are used in an increasing array of applications [1]. Conventional SEM is often used to study their morphology [2, 3]. However, since they are used when hydrated, cryo-SEM is a superior tool to study microgel morphology. Here, we apply cryo-SEM to study hydrated microgels of poly(ethylene glycol)-co-(acrylic acid) (PEG-AA).

Plunge freezing is a common method to prepare hydrated samples for cryo-SEM. Thin specimens are rapidly immersed in liquid nitrogen and cryo-transferred to the cryo-SEM. Fig. 1a shows an example from a cryo-plunged aqueous colloidal solution of PEG-AA microgels. While a hydrated microgel should have a smooth surface due to surface-tension effects, fig. 1a shows microgels with rough surfaces. Roughness is presumably due to microgel reorganization during water crystallization. In contrast, Fig. 1b shows an identical specimen prepared by high-pressure freezing (Leica HPM-100 HPF). The HPF method gives rise to frozen-hydrated microgels each having a very smooth surface. HPF is more effective in freezing water to its amorphous state and thus preserves the intrinsic microgel morphology. Similar effects occur during the cryo-preparation of thick bacterial biofilms [4].

Specimens were cryo-fractured after HPF. By warming the specimen to $-95^{\circ} \mathrm{C}$ for $10 \mathrm{~min}$, approximately $1 \square \mathrm{m}$ of water was removed by sublimation from the fracture surface (Fig. 2). Residual polymer and unreacted monomer in the solution surrounding the microgels becomes apparent upon sublimation. Importantly, microgels can bind water strongly. In the case of PEG-AA (10 wt $\%$ EG : 1 wt\% AA) microgels, cryo-SEM suggests that the sublimation rate from the frozen-hydrated microgels is significantly lower than that from the surrounding embedding ice. EDS X-ray spectroscopy in the cryoSEM confirmed the presence of bound water within the microgels despite the significant sublimation of the surrounding ice. Since PEG-AA microgels consist of C, H, and O, we can follow the microgel water content by monitoring the C/O EDS intensity ratio. To minimize specimen charging during imaging, we used $1 \mathrm{keV}$ electrons, and these are sufficient to excite both $\mathrm{C}$ and $\mathrm{O} \mathrm{K} \mathrm{X}$-rays. $1 \mathrm{keV}$ incident electrons furthermore have limited electron penetration into the specimen, thus ensuring that X-rays are generated very locally from an individual microgel. Fig. 3 shows a typical result. EDS measurements from microgels after mild sublimation $(\sim 1 \square \mathrm{m}$ bulk water removed) and strong sublimation ( $\sim 5 \mathrm{~m}$ bulk water removed) find almost identical $\mathrm{C} / \mathrm{O}$ intensity ratios (Table 1). This result suggests that water is tightly bound within these microgels, presumably because of strong dipole interactions with the PEG ether oxygens. [5]

\section{References:}

[1] Martin Malmsten, Helena Bysell and Per Hansson, Current Opinion in Colloid \& Interface Science 15 (2010), p. 435.

[2] B.G. De Geest, C. Déjugnat and et al. Journal of Controlled Release 116 (2006), p. 159.

[3] Antonio Fernández-Barbero et al. Advances in Colloid and Interface Science, 147-148 (2009), p. 88.

[4] Yong Wu et al., Microscopy and Microanalysis, Vol 20 Issue 5 (2014), p.1348 
[5] This project was supported by the U.S. Army Research Office via grant W911NF-12-1-0331 using microscope resources partially funded by the NSF via grants DMR-0922522 and DMR-1428296.
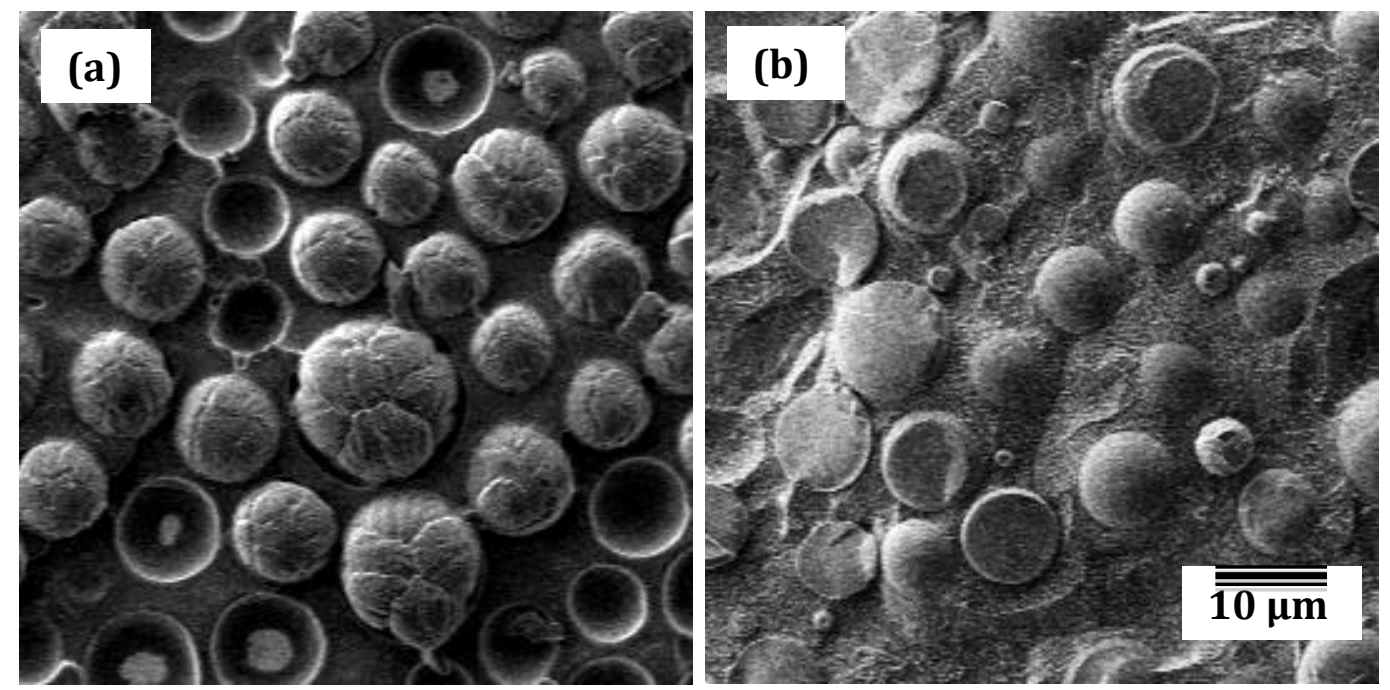

Figure 1. SEM micrograph of PEG-AA microgel prepared by (a) plunge freezing and (b) HPF.

(a)
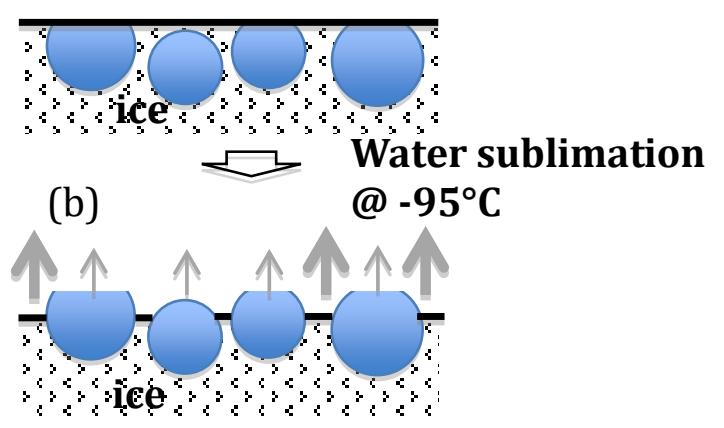

\begin{tabular}{|l|c|c|c|}
\hline & C counts & O counts & $\begin{array}{l}\text { C/O count } \\
\text { ratio }\end{array}$ \\
\hline $\begin{array}{l}\text { Mild sublimed } \\
\text { ( 1um ice removed) }\end{array}$ & 1226 & 2764 & 0.44 \\
\hline $\begin{array}{l}\text { Strong sublimed } \\
\text { ( 5um ice removed) }\end{array}$ & 731 & 1681 & 0.43 \\
\hline Freeze dried & 12071 & 336 & 35.92 \\
\hline
\end{tabular}

Table 1. EDS measurement of the sample C/O count ratio

Figure 2. (a) as fractured cryo-sample.

(b) sample sublimed at $-95^{\circ} \mathrm{C}$.
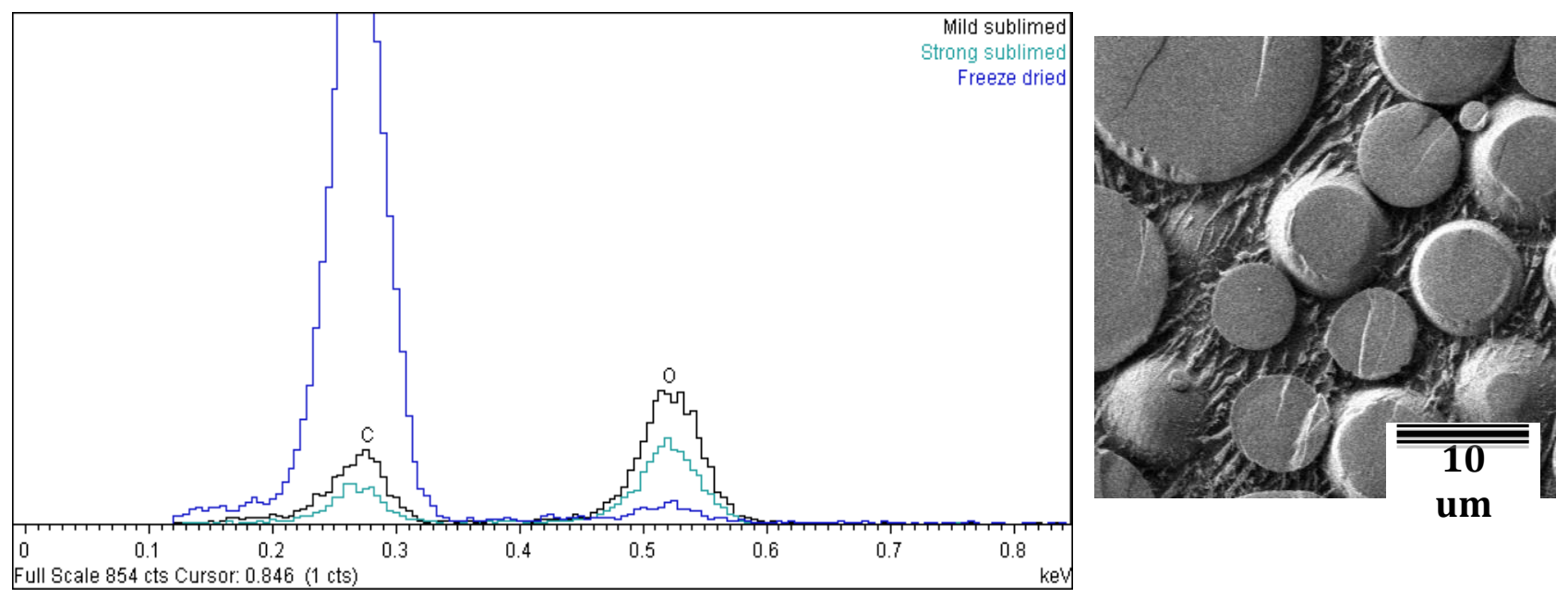

Figure 3. EDS spectra of mild sublimed gel sample. Approximately 1um of water removed from the surface.
the 